LA-UR-01-3471

Approved for public release: distribution is unlimited.

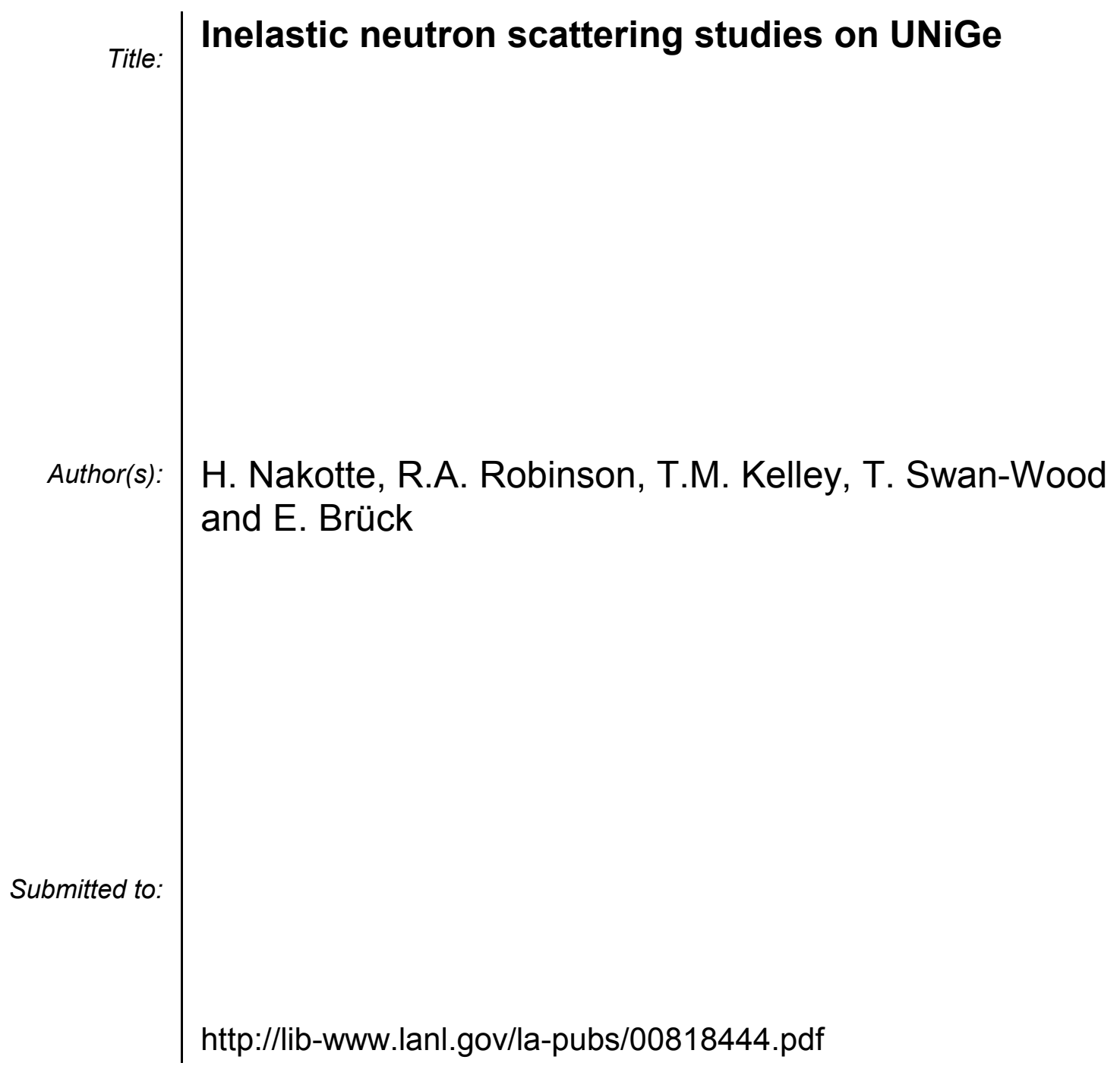

Los Alamos National Laboratory, an affirmative action/equal opportunity employer, is operated by the University of California for the U.S. Department of Energy under contract W-7405-ENG-36. By acceptance of this article, the publisher recognizes that the U.S. Government retains a nonexclusive, royaltyfree license to publish or reproduce the published form of this contribution, or to allow others to do so, for U.S. Government purposes. Los Alamos National Laboratory requests that the publisher identify this article as work performed under the auspices of the U.S. Department of Energy. Los Alamos National Laboratory strongly supports academic freedom and a researcher's right to publish; as an institution, however, the Laboratory does not endorse the viewpoint of a publication or guarantee its technical correctness. 
SCES 2001 paper: 182

Version: June 14, 2001

\title{
Inelastic neutron scattering studies on UNiGe
}

H. Nakotte ${ }^{\mathrm{a}, \mathrm{b}}$, R.A. Robinson ${ }^{\mathrm{b}, *}$, T.M. Kelley ${ }^{\mathrm{b}, \mathrm{c}}$,

T. Swan-Wood ${ }^{\text {b*** }}$ and E. Brück ${ }^{\mathrm{d}}$

${ }^{a}$ Physics Department, New Mexico State University,

Las Cruces NM 88003

${ }^{b}$ LANSCE-12, Los Alamos National Laboratory,

Los Alamos NM 87545

${ }^{c}$ Department of Physics, University of California, Riverside CA 92521

${ }^{d}$ Van der Waals-Zeeman Instituut, Valckenierstraat 65,

Universiteit van Amsterdam, 1018 XE Amsterdam,

The Netherlands

\begin{abstract}
We measured the inelastic neutron scattering response of $\mathrm{UNiGe}$ at various temperatures using incident beam energies of 25 and $100 \mathrm{meV}$. Below $\mathrm{T}_{\mathrm{N}}=$ $50 \mathrm{~K}$, there is evidence for the formation of a gap as indicated by reduced scattering close to the elastic peak. Furthermore, we find an excitation around $30 \mathrm{meV}$ in addition to the phonon background for temperatures below $130 \mathrm{~K}$. The results are discussed in terms of spin waves with formation of a gap in the excitation spectrum below $\mathrm{T}_{\mathrm{N}}$.
\end{abstract}

Keywords: inelastic neutron scattering, spin waves, uranium compounds

\section{Corresponding author:}

Heinz Nakotte

Physics Department

New Mexico State University

Las Cruces NM 88003

Phone: 1-505-646-2459

Fax: 1-505-646-1934

e-mail: hnakotte@nmsu.edu 
A very prominent parameter for the magnetism in uranium compounds is strong $5 f$-ligand hybridization, which is believed to be the origin of the magnetic anisotropy in these materials [1]. Here, we consider the case of UNiGe, where the magnetic anisotropy within the $b-c$ plane is much weaker compared to the $a$-axis anisotropy. UNiGe undergoes two antiferromagnetic transitions at around $50 \mathrm{~K}$ (incommensurate magnetic order) and at $42 \mathrm{~K}$ (commensurate order) [2].

Considering the possible influence of low-energy magnetic excitations on the bulk properties of a material leads to the concept of spin waves. Having in mind the strong magnetic anisotropy in uranium compounds, we may expect a gap in the energy spectrum of such excitations. In the planaranisotropy case, where the in-plane anisotropy is 'small' (like UNiGe), one expects to observe spin waves or similar excitations with an energy gap $\Delta$, which will give rise to an exponential term due to electron-magnon scattering in the temperature dependencies of the electrical resistivity and the specific heat [3]. Bulk investigations indeed revealed such exponential contributions in UNiGe with an energy gap $\Delta=40 \mathrm{~K}$ [4].

We performed inelastic-neutron-scattering studies using the inelastic spectrometer PHAROS at the Manuel Lujan jr. Neutron Scattering Center, Los Alamos National Laboratory, to test the possibility of spin waves in UNiGe. The experiments were done on 30 gram of polycrystalline UNiGe at seven different temperatures between 26 and $150 \mathrm{~K}$ using incident beam energies of 25 and $100 \mathrm{meV}$. Data were taken using a low-angle detector bank, which did not allow for any dispersion study because of the limited $\mathbf{Q}$ coverage.

In Fig. 1, we display the inelastic-scattering response with $E_{i}=100$ $\mathrm{meV}$ at four different temperatures: $26 \mathrm{~K}$ (commensurate phase) and $45 \mathrm{~K}$ (incommensurate phase) as well as $100 \mathrm{~K}$ and $150 \mathrm{~K}$ (paramagnetic phase). There is clear evidence for additional quasielastic scattering, in particular at higher temperatures. It has been argued that in uranium compounds there may be contributions from quasielastic scattering because of hybridization of the $5 \mathrm{f}$ electrons with the conduction electrons [5]. Quasielastic scattering is found also at lower temperatures, but reduced scattering indicates the formation of a 'hybridization gap' below $\mathrm{T}_{\mathrm{N}}$. Keeping in mind the intimate connection between $5 f$-ligand hybridization and magnetism, such gap may cause subsequent gapping in the magnon-excitation spectrum. Using $E_{i}=25$ $\mathrm{meV}$ (not shown), we were able to determine that the size of the gap is about 
$6 \mathrm{meV}(\approx 70 \mathrm{~K})$, which is somewhat larger than the values for $\Delta$ from bulk measurements.

In addition, we find a clear excitation at $30 \mathrm{meV}$ at low temperatures. As can be seen in Fig. 2, there seems to be little dependence of its location with temperature, while the integrated intensity decreases with increasing temperature until it is barely visible at $\mathrm{T}=150 \mathrm{~K}$. This behavior excludes that the excitation is of phonon-origin, thus it is magnetic. The observed temperature dependence of the excitation is inconsistent with the one expected for a simple single-ion crystal-field excitation, thus we speculate that it is due to spin waves. However, spin waves are rarely observed for temperatures above the ordering temperature, and in UNiGe the excitation persists up to $120 \mathrm{~K}$ (more than twice the value of $\mathrm{T}_{\mathrm{N}}$ ). On the other hand, spin waves far above the ordering temperature have been established in some low-dimensional systems [6], and similar behavior may not be inconceivable in uranium compounds due to their large magnetic anisotropy. In summary, we found some evidence for spin waves in UNiGe. Spin waves exist already at temperatures substantially above $T_{N}$, and a gap in the magnon-excitation spectrum forms at $\mathrm{T}_{\mathrm{N}}$. Confirmation of the above picture will require a detailed analysis of the dispersion curves using singlecrystalline UNiGe. Since $5 f$-ligand hybridization is expected to contribute to the spin-wave stiffness, this would provide some insight how spin waves propagate in systems with essentially delocalized $5 f$ moments. 


\section{References:}

*present address: ANSTO, PMB1, Menai, NSW 2234, Australia

**present address: Keck Laboratory, California Institute of Technology, Pasadena CA 91125

[1] V. Sechovksy and L. Havela, in Ferromagnetic Materials, Vol. 11, ed. K.H.J. Buschow, 1998.

[2] H. Nakotte, A. Purwanto, R.A. Robinson, Z. Tun, K. Prokes, A.C. Larson, L. Havela, V. Sechovsky and H. Maletta, Phys. Rev. B 54 (1996) 7201

[3] N.H. Andersen and H. Smith, Phys. Rev. B 19 (1979) 384

[4] L. Havela, V. Sechovsky, H. Nakotte, A. Lacerda, K. Prokes and E. Brück, Czech. J. Phys. 46 (1996) 2043

[5] A. Hiess, L. Havela, K. Prokes, R.S. Eccleston and G.H. Lander, Physica B 230-232 (1997) 89

[6] R.A. Cowley, D.A. Tennant, S.E. Nagler and A.M. Tsvelik, Phys. Rev. B 52 (1995) 13368 


\section{Figure Captions:}

Fig. 1: Inelastic-scattering response of UNiGe using 100-meV incident energy at four different temperatures. The solid lines represent Gaussian fits of the 30-meV excitation after subtraction of the background, the elastic and the quasielastic contributions.

Fig. 2: Temperature dependence of a) the position of the excitation and b) the integrated intensity of the $30-\mathrm{meV}$ excitation in UNiGe. 

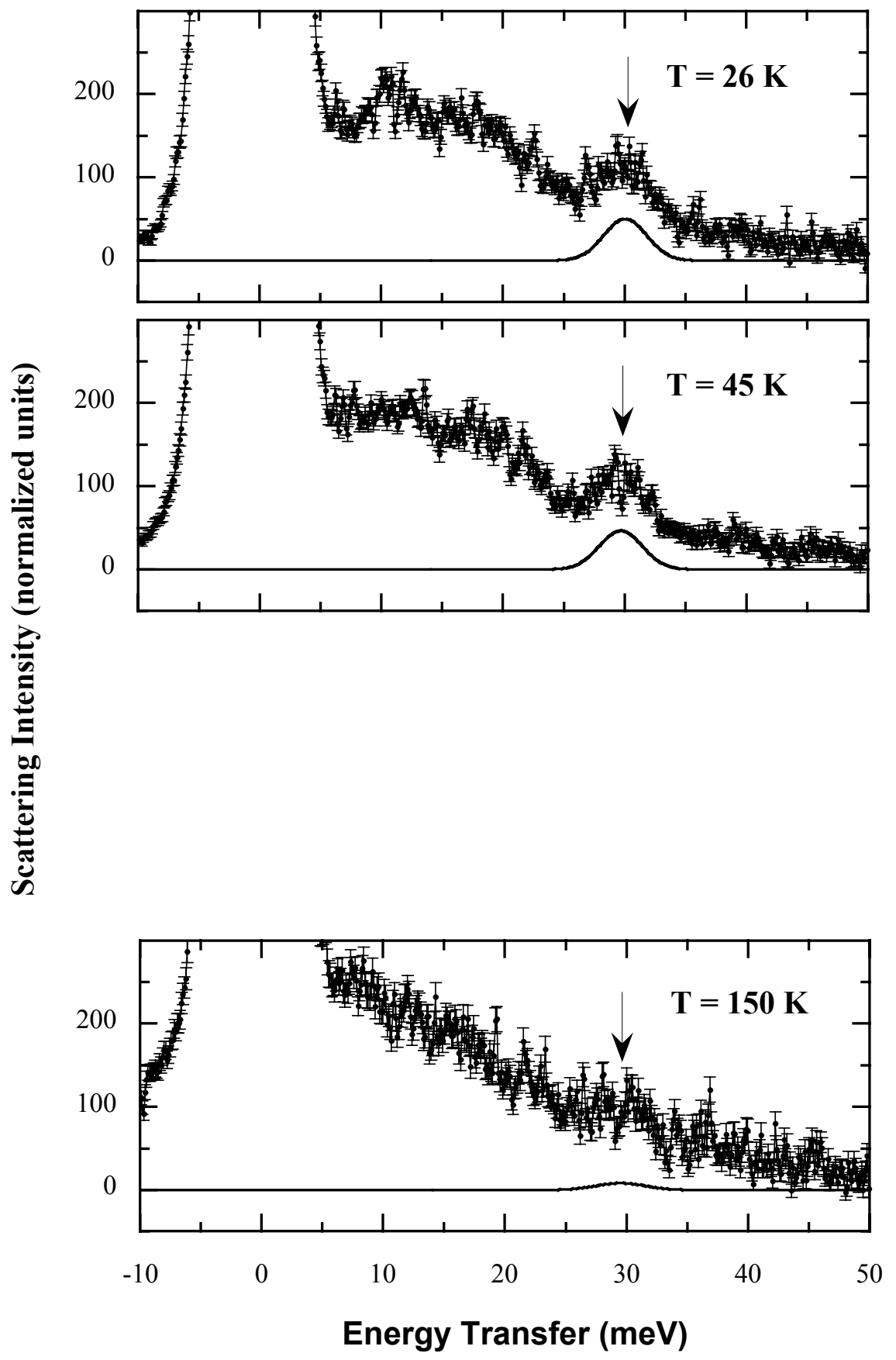

Fig. 1: H. Nakotte et al., Inelastic neutron scattering studies on UNiGe 


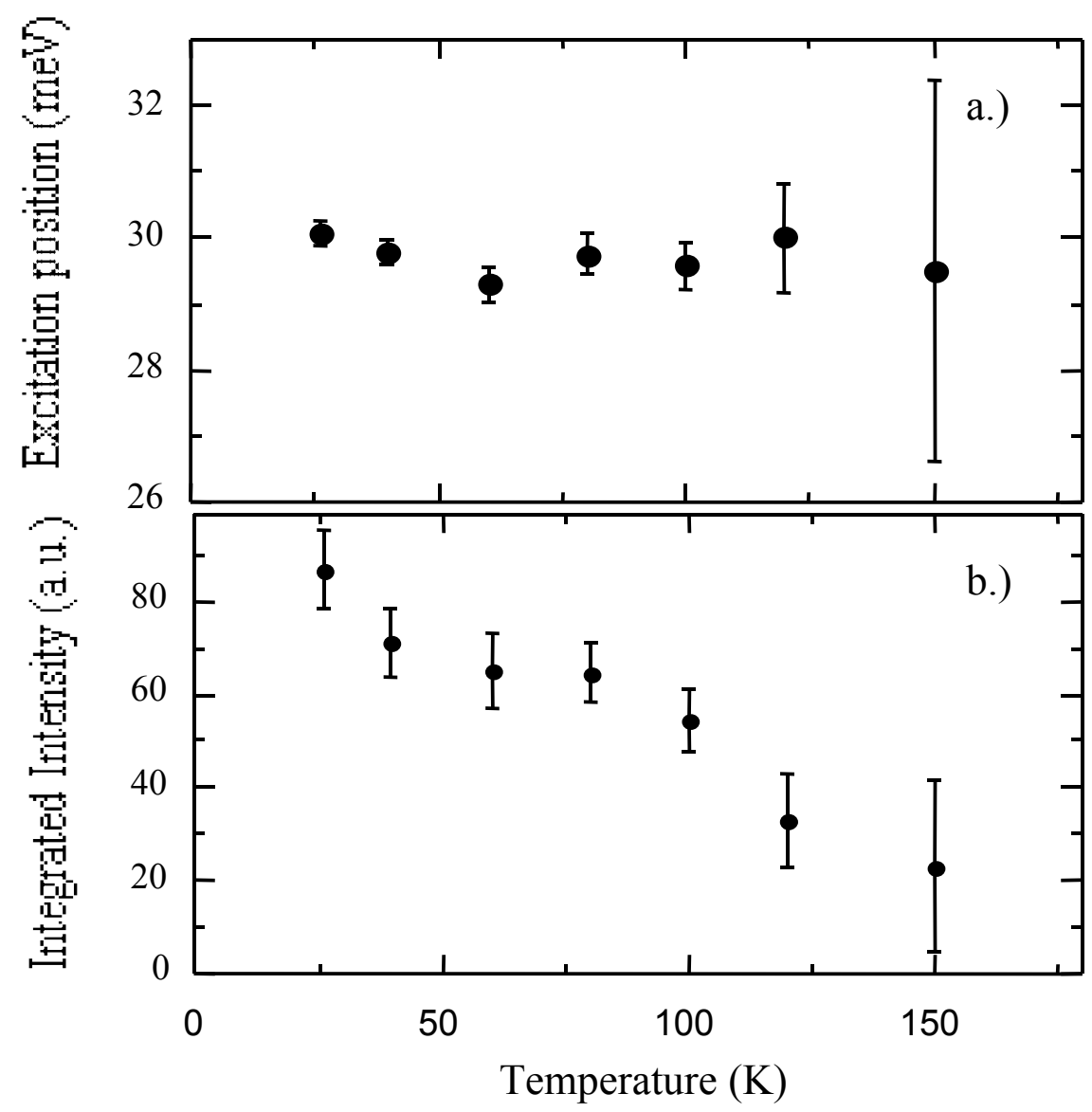

Fig. 2: H. Nakotte et al., Inelastic neutron scattering studies on UNiGe 